\title{
Interactive Boundary-Layer Method for Unsteady Airfoil Flows: Quasisteady Model
}

\author{
Tuncer Cebeci* \\ University of Michigan, Ann Arbor, Michigan \\ and \\ Hong-Ming Jang $\dagger$ \\ Douglas Aircraft Company, Long Beach, California 90846
}

\begin{abstract}
An interactive boundary-layer method previously developed and tested for steady flows is used here in a quasisteady manner to examine the evolution of the flow behavior of airfoils subject to harmonic oscillation and ramp-type motions. The calculations encompass the airfoil and wake flows at angles of attack that lead to separation. The results quantify the effects of the viscous boundary layer and wake on the variation of lift coefficient with angle of attack and reduced frequency. These effects are shown to be large at angles of attack that involve boundary-layer separation.
\end{abstract}

\section{Introduction}

T HE effect of unsteady motion of an airfoil on its stall behavior is of considerable interest to many practical applications including the blades of helicopter rotors and of axial compressors and turbines.' Experiments with oscillating airfoils, for example, have shown that the flow can remain attached for angles of attack greater than those that would cause stall to occur in a stationary system. This result appears to stem from the formation of a vortex close to the surface of the airfoil that continues to provide lift. It is also evident that the onset of dynamic stall depends strongly on the airfoil section, and as a result great care is required in the development of a calculation method that will accurately predict this behavior.

In principle, the prediction of dynamic stall can be accomplished by solving the Reynolds-averaged Navier-Stokes equations or their reduced forms. A turbulence model is required and is presumed, with reasonable supporting evidence (see Ref. 2), to be uninfluenced by the imposed unsteadiness. Several papers have been prepared with calculations of this type (see for example Ref. 3) and involve the solution of equations with two diffusion terms as well as parabolized forms and thin-layer approximations. An alternative is to make use of interactive boundary-layer theory whereby inviscid and boundary-layer equations are solved and allowed to influence each other by an iterative scheme.

Extensive investigations with an interactive approach have been reported by Cebeci et al. ${ }^{4}$ and show that the incompressible flow and performance characteristics of airfoils can be predicted accurately and efficiently for high and low Reynolds numbers and for a range of angles of attack up to and including stall. At incidence angles higher than stall, however, this procedure was unable to predict the airfoil performance due to relatively large regions of flow separation on the surface and in the wake. Near stall, the value of the trailing-edge displacement thickness approached $10 \%$ of the chord, and the numerical method could not provide converged solutions. The predictions of this interactive boundary layer are similar to those

Presented as Paper 89-0020 at the AIAA 27th Aerospace Sciences Meeting, Reno, NV, Jan. 9-12, 1989; received March 20, 1989; revision received Jan. 12, 1990. Copyright (C) 1989 American Institute of Aeronautics and Astronautics. All rights reserved.

*MDC Senior Fellow. AIAA Fellow.

†Graduate Student, Mechanical Engineering Department. Member AIAA. obtained from solutions of thin-layer Navier-Stokes by the ARC-2D method ${ }^{5,6}$ for angles of attack up to and including stall (see Fig. 1). It has been shown in Ref. 4 that the interactive flow calculations, without the wake effect and for angles of attack greater than that of stall, yielded lift coefficients that increased with incidence angle almost in the same way as those computed with the thin-layer Navier-Stokes approach with the wake effect included. When the wake effect was included in the interactive boundary-layer calculations, the results agreed more closely with measurements but could not be extended beyond the stall angle.

More recently, the interactive method has been improved to permit calculations for angles of attack greater than that of stall, and the results have been shown to have the correct behavior. To achieve this, modifications were made to the iterative procedure and to the method of calculating the wake. These improvements are described in Ref. 8 and are necessary where results are required at angles of attack corresponding to stall and poststall.

The interactive boundary-layer method for steady flows has also been extended to study the laminar separation and reattachment near the leading edge of a thin oscillating airfoil, ${ }^{9}$ but the calculation of flow over practical airfoils involves laminar and turbulent flows, and the inclusion of the upstream influence of the wake requires careful step-by-step development and evaluation, as has been done for steady flows. The use of a quasisteady approach to unsteady flows thus represents an essential building block in a progression toward an interactive calculation method that solves unsteady equations even though the latter is likely to represent a much wider range of oscillation frequencies. The extent of the differences can be quantified only by comparing results from both. The present study permits assessment of a quasisteady method over a range of angles of attack and frequency in terms of convenience, accuracy, and computational cost. It should be remembered that the main purpose of boundary-layer calculations is to include the viscous effects in the inviscid method, and, since this is achieved in an iterative manner, it is useful to use a quasisteady approach in the early stages of the unsteady flow calculations. This strategy can improve the efficiency of the calculations, reduce the computer time, and avoid possible difficulties that may arise when the unsteady boundary-layer equations are solved.

The calculation of the wake flow becomes more difficult with increasing angle of attack, and investigations of steady flows indicate that near and past stall, the behavior of the velocity profile at the trailing edge resembles that of a mixing layer with a huge backflow. The thickness of the upper velocity 


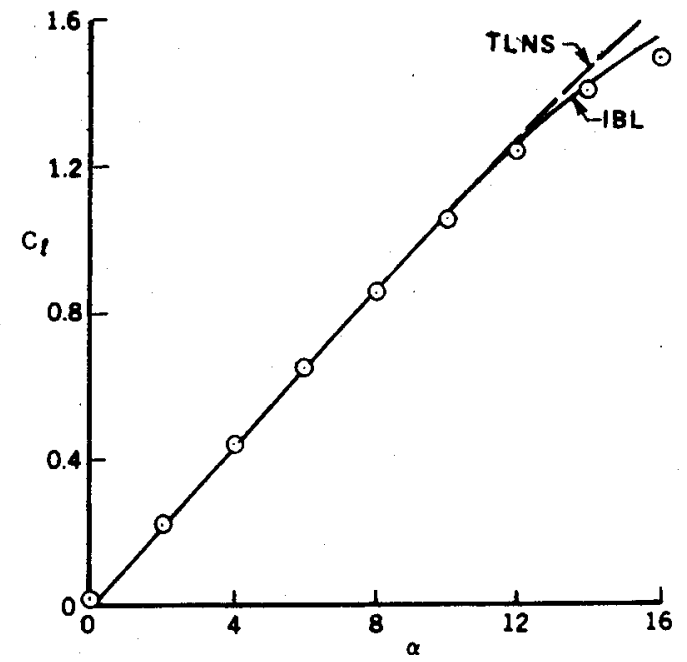

Fig. 1 Predictions of the thin-layer Navier-Stokes (TLNS) and interactive boundary-layer (IBL) methods for the NACA 0012 airfoil; symbols denote experimental data of Ref. 7 .

profile is an order of magnitude larger than the lower velocity profile, and the upper velocity profile contains flow reversal, which increases with angle of attack, whereas the lower surface velocity profile is almost laminar-like and has no flow reversal. The FLARE approximation, in which the convective term $u$ $\partial u / \partial x$ in the $x$-momentum equation is set equal to zero in regions of negative velocity, has yet to be evaluated for wakes of this type, and indeed, the only real test of the FLARE approximation, first suggested by Reyhner and Flügge-Lotz, ${ }^{9}$ is that of Cebeci et al. ${ }^{7}$ for steady flows. For these reasons, a useful additional step towards the long-term objective of fully unsteady-flow calculations is to solve the boundary-layer equations in time-dependent form on the airfoil and in quasisteady form in the wake.

The purpose of this paper is to present calculations of unsteady flows obtained with the quasisteady approach and to assess the range of conditions for which this approach can be expected to provide useful results. The interactive boundarylayer method is described in the following section and the results presented and discussed in Sec. III. The paper ends with a summary of the more important conclusions.

\section{Interactive Boundary-Layer Method}

The unsteady interactive boundary-layer method makes use of the panel method developed by Platzer and his student Teng ${ }^{11}$ and the inverse boundary-layer method of Cebeci et al. ${ }^{4}$ The solution of the inviscid flow equations for the airfoil is similar to that of Hess and Smith ${ }^{12}$ and uses the procedure of Basu and Hancock ${ }^{13}$ for the wake. The shape of the airfoil is represented by a large number of straight-line segments called panels (an inscribed polygon), each of which has a different constant source strength. The wake is represented by a series of free vortices shed from the trailing edge, each having a constant vorticity strength whose variation along the wake is determined by the time history of the circulation about the airfoil.

The boundary-layer method is based on the solution of the two-dimensional, steady, boundary-layer equations expressed in terms of an eddy viscosity $\epsilon_{m}$ so that the continuity and momentum equations have the form

$$
\begin{gathered}
\frac{\partial u}{\partial x}+\frac{\partial v}{\partial y}=0 \\
u \frac{\partial u}{\partial x}+v \frac{\partial u}{\partial y}=u_{e} \frac{\partial u_{e}}{\partial x}+\frac{\partial}{\partial y}\left[\left(v+\epsilon_{m}\right) \frac{\partial u}{\partial y}\right]
\end{gathered}
$$

and are subject to boundary conditions

$$
y=0, \quad u=v=0, \quad y \rightarrow \infty, \quad u \rightarrow u_{e}(x)
$$

which include the flow on the airfoil. With $y=0$ now denoting the dividing streamline that separates the upper and lower parts of the inviscid flow in the wake, they can be written as

$$
y \rightarrow \infty, u \rightarrow u_{e}(x) ; \quad y=0, v=0 ; \quad y \rightarrow-\infty, u \rightarrow u_{e}(x)
$$

In order to avoid the breakdown of the solutions at flow separation, the outer boundary condition expresses $u_{e}(x)$ as the sum of inviscid velocity $u_{e}^{0}(x)$ and a perturbation velocity $\delta u_{e}(x)$ computed from the Hilbert integral

$$
\delta u_{e}(x)=\frac{1}{\pi} \int_{x_{a}}^{x_{b}} \frac{\mathrm{d}}{\mathrm{d} \sigma}\left(u_{e} \delta^{*}\right) \frac{\mathrm{d} \sigma}{x-\sigma}
$$

with the interaction scheme confined to $\left(x_{a}, x_{b}\right)$. Introduction of a discrete approximation to this integral enables the perturbation velocity to be expressed in terms of the geometric coefficients of the airfoil $c_{i j}$ and enables the edge boundary condition to be written in a form in which a relationship is provided with the external velocity and displacement thickness, that is,

$$
u_{e}(x)=u_{e}^{0}(x)+\sum_{j=1}^{n} c_{i j}\left(u_{e} \delta^{*}\right)_{j}
$$

and can be generalized to the form

$$
u_{e}(x)=u_{e}^{\kappa}(x)+\sum_{j=1}^{n} c_{i j}\left(D_{j}-D_{j}^{\kappa}\right)
$$

where $D=u_{e} \delta^{*}$ and $u_{e}^{\kappa}$ correspond to the inviscid velocity distribution that contains the displacement thickness effect $\left(\delta^{*}\right)$ computed from a previous sweep, as discussed next. This expression was suggested by Veldman for steady flows ${ }^{14}$ and can also be viewed as an empirical formula that provides a better approximation to the link between inviscid and viscous flow equations than the relaxation formula used by LeBalleur ${ }^{15}$ and Carter. ${ }^{16}$

The solution of the system given in Eqs. (1-4), with the outer edge specified by Eq. (5) and with the algebraic eddy viscosity of Cebeci and Smith, is obtained by Keller's two-point, finitedifference method described in Ref. 4. For a given pressure distribution and chord Reynolds number $R_{c}\left(\equiv u_{\infty} c / \nu\right)$ the boundary-layer calculations start at the stagnation point and are performed for a laminar flow in the direct mode for a few $x$ stations, after which they are performed in the inverse mode. Turbulent flow calculations begin at the transition location, which is specified at the pressure peak or computed from formulas such as that of Michel, ${ }^{17}$ and extend into the wake. The calculated displacement thickness and external velocity distribution are used to determine a blowing velocity distribution $v_{n}=\mathrm{d} D / \mathrm{d} s$ so that the inviscid flow equations are solved again subject to a new boundary condition. The inviscid velocity distribution is evaluated directly on the displacement surface, whereas the blowing velocity is applied on the original airfoil surface. The Kutta condition is also satisfied at the displacement surface, and this process is repeated on an iterative basis until the solutions of both boundary-layer and inviscid-flow equations converge.

This procedure is appropriate as long as there is no separation on the airfoil. With separation, the FLARE approximation was used and proved to be satisfactory when the separation region was small. As the extent of the separation region on the airfoil increased with increasing angle of attack, an additional iterative scheme, based on a homotopy continuation method $^{4}$ was introduced at the start of the wake calculations. Thus, an initial velocity profile was defined by

$$
u=u_{\mathrm{ref}}+n\left(u_{a}-u_{\mathrm{ref}}\right)
$$

and the boundary-layer equations solved at the first $x$ station 
on the wake with $n=0$. Here $u_{\text {ref }}$ corresponds to a nonseparating velocity profile constructed arbitrarily from the separated velocity profile at the previous station that has the velocity profile denoted by $u_{a}$. The calculation was then repeated for different values until the solutions converged. This procedure was applied for each velocity profile in the wake with separation and was necessary when the extent of the trailing-edge separation was around $10 \%$ of the chord; it resulted in successful calculations of the lift and drag coefficients of several airfoils up to and including stall. With the improvements described in Ref. 8 , it can now be extended to compute poststall flows on airfoils.

\section{Results and Discussion}

The method described in the previous section, in which the inviscid flowfield is computed by the unsteady panel method of Ref. 11 and the viscous flowfield by the steady inverse boundary-layer method of Ref. 4, was used to compute flow over a Sikorsky SSC-A09 airfoil subject to a harmonic oscillation and ramp-type motion with constant pitch rate. At first, calculations were performed for steady flows so that the results could be compared with experimental data for a range of angles of attack including poststall, as described in IIIA below. Next, calculations described in IIIB were performed with the quasisteady approach by interacting the unsteady-flow panel method with the inverse steady boundary-layer method. This step removed the potential difficulties of the unsteady-flow boundary layer and allowed emphasis on the unsteady-flow panel method. The quasisteady model should, of course, be able to simulate flows over airfoils executing slow motion and will overlap the final work of unsteady flow calculations. The conditions for which quasisteady flow calculations are appropriate are

$$
\frac{\partial u}{\partial t} \text { and } \frac{\partial u_{e}}{\partial t} \ll u_{e} \frac{\partial u_{e}}{\partial x}
$$

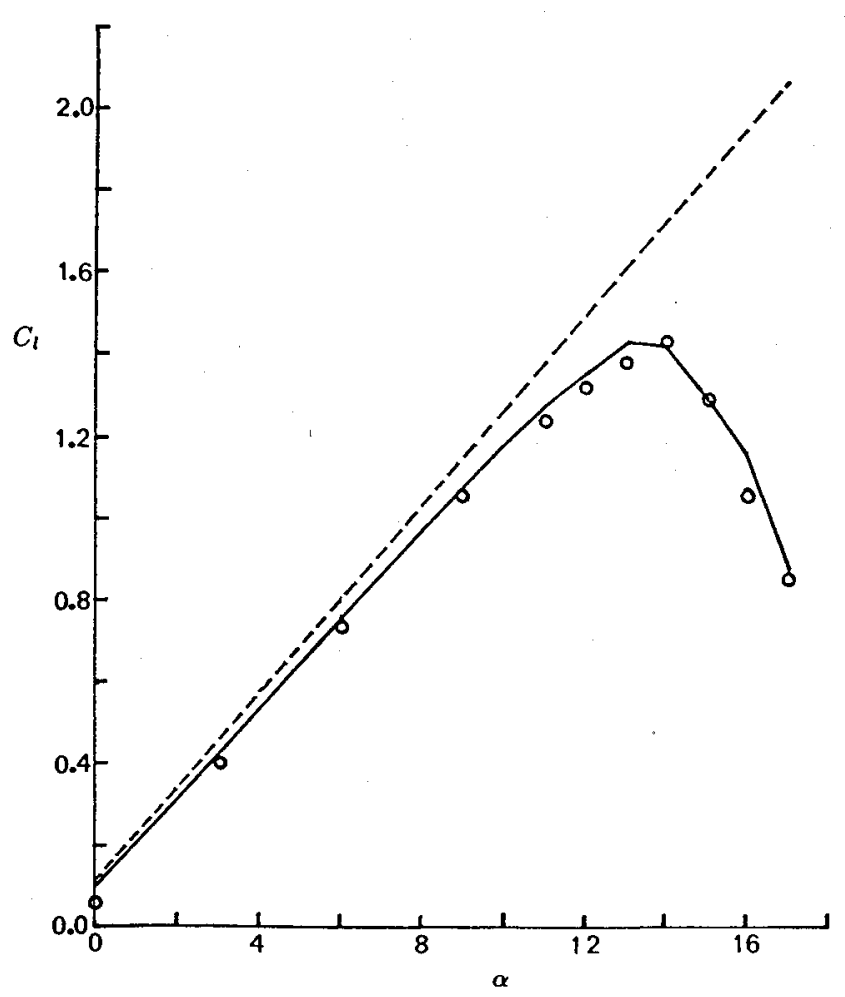

Fig. 2 Variation of lift coefficient with angle of attack for steady flow over the Sikorsky airfoil at $R_{c}=2 \times 10^{6}$, --- panel and - interactive methods, o data of Ref. 18. a)
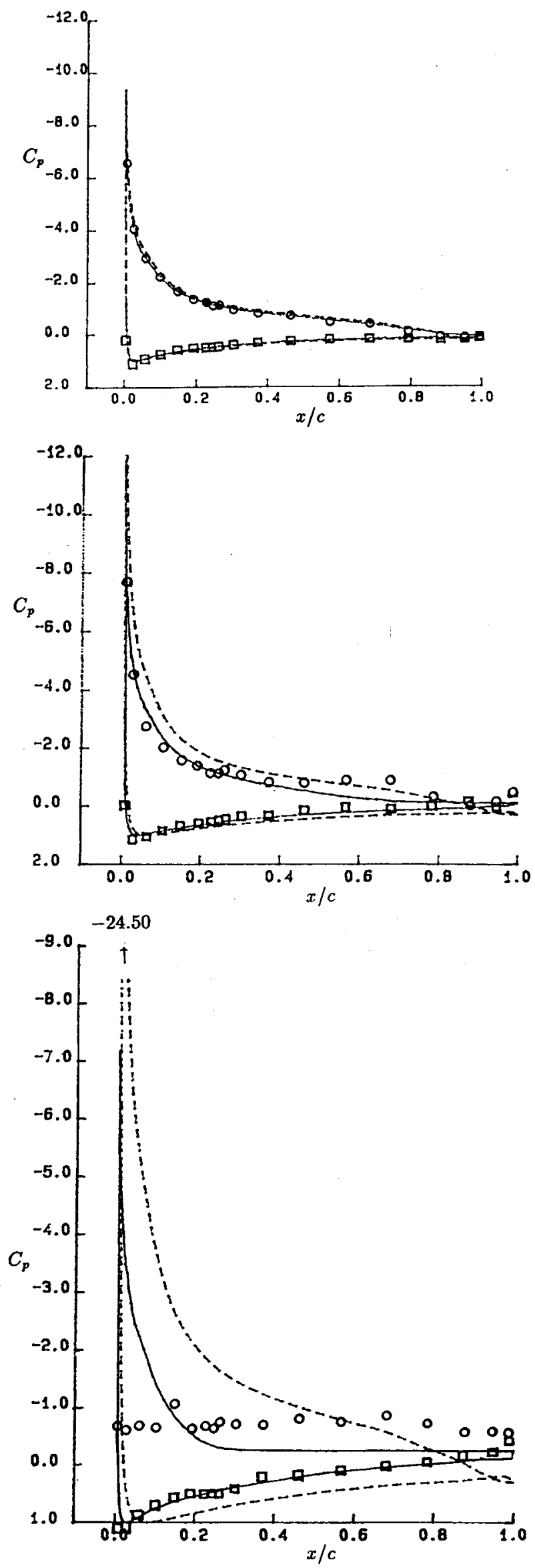

Fig. 3 Pressure-coefficient variations for the Sikorsky airfoil at $R_{c}=2 \times 10^{6}:$ a) $\alpha=11 \mathrm{deg}$; b) $\alpha=15 \mathrm{deg}$; c) $\alpha=17 \mathrm{deg}$;-.. panel and - interactive methods; $o$ is the upper surface and $\square$ the lower surface in the experiments of Ref. 18. 

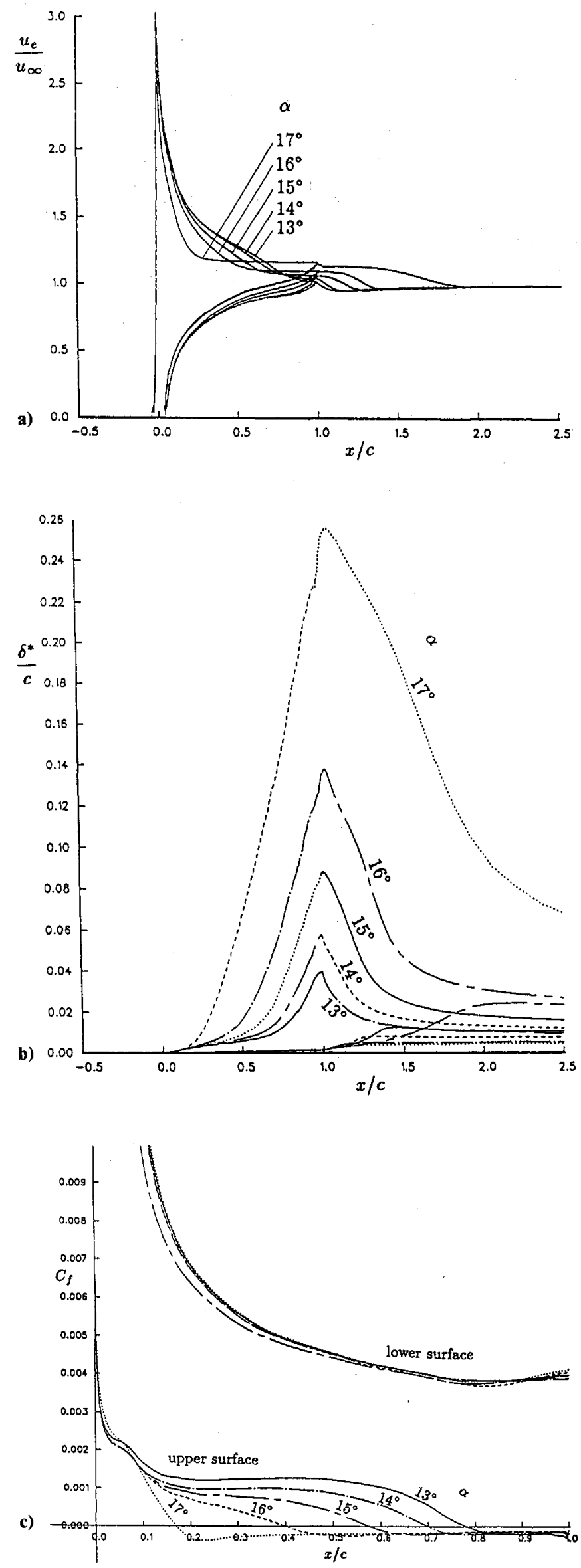

Fig. 4 Calculated variations: a) external velocity; b) displacement thickness; and c) local skin-friction coefficient distributions for the Sikorsky airfoil at $R_{c}=2 \times 10^{6}$.

\section{A. Steady Flow}

Calculations were performed for steady flow over the Sikorsky airfoil at a chord Reynolds number of $2 \times 10^{6}$ with the location of transition computed from Michel's formula, except where the boundary-layer separated upstream of this location, in which case transition was assumed to occur at the separation point. At higher angles of attack, separation occurred at a very short distance from the pressure peak, and in those cases, transition was assumed to occur at a very small distance upstream of the pressure peak. This procedure allowed the calculations to be performed for a range of angles of attack, including poststall, and to be compared with the measurements of Lorber and Carta. ${ }^{18}$

Figures 2, 3, and 4 show the variations of lift coefficient, pressure coefficient, and boundary-layer parameters as a function of angle of attack and confirm that the interactive method is able to represent measurements for angles up to and beyond that of stall. The close agreement between the calculated lift coefficients and the measurements of Ref. 18 are in contrast to the solutions of the inviscid-flow equations that, as expected, increase linearly with angle and do not recognize that the airfoil has stalled. The pressure-coefficient distributions of Fig. 3 reveal the same features with the inviscid and interactive calculations providing similar results at angles of attack below 11 deg and increasingly different results as the angle of attack increases. Again, the interactive results are in close accord with measurements except perhaps at $17 \mathrm{deg}$ where the large region of upper-surface separation introduces inaccuracies in measurements and calculations.

The calculated boundary-layer properties of Fig. 4 show the expected large variations as angle of attack increases to and beyond stall. The variations of $u_{e} / u_{\infty}$ are strongly dependent on the angle of attack, particularly in the trailing-edge region and correctly tend to unity with increasing $x$. The displacement thickness on the upper surface achieves very high values and on the airfoil is always very much larger than that on the lower surface. There is also a tendency for the location of the displacement-thickness maximum to move from the trailing edge downstream with angle of attack, and for 17 deg it occurs at $x=1.07$. The skin-friction coefficient confirms that the location of boundary-layer separation moved upstream with angle of attack and that there was attached flow over only $0.17 \mathrm{c}$ for the 17-deg angle. The much higher values of $c_{f}$ of the lowersurface boundary layer are evident.

\section{B. Quasisteady Flow}

Having demonstrated that the steady flow over the Sikorsky airfoil can be predicted accurately for all angles of attack including poststall, calculations were performed with the quasisteady approach. At first, and to check the validity of the quasisteady model, two types of slow motion corresponding to a harmonic oscillation according to

$$
\alpha(t)=5 \operatorname{deg}+10 \mathrm{deg} \sin \omega t
$$

at a reduced frequency $\omega$ of $10^{-5}$ and a ramp-type motion from 0 to $16 \mathrm{deg}$ at constant pitch $A\left(\equiv d \alpha / d t c / V_{\infty}\right)$ of $10^{-5}$ were considered for the same airfoil at a Reynolds number $2 \times 10^{6}$. Under these conditions the maximum angle of attack exceeded the static stall angle in each case, and since the influence of the wake is known to be important, particular attention was directed to its correct representation. Subsequently, calculations were performed for higher frequencies to determine the unsteady effects.

The variations of lift coefficient, pressure coefficient, and boundary-layer parameters were examined for angles of attack up to $15 \mathrm{deg}$ and proved to be identical to those of Figs. 2, 3, and 4 . Thus, the quasisteady-flow calculation method performed correctly and without numerical difficulties. Figure 5 shows the location of the stagnation point and of the displacement thickness at the trailing edge as functions of time, and 
a)

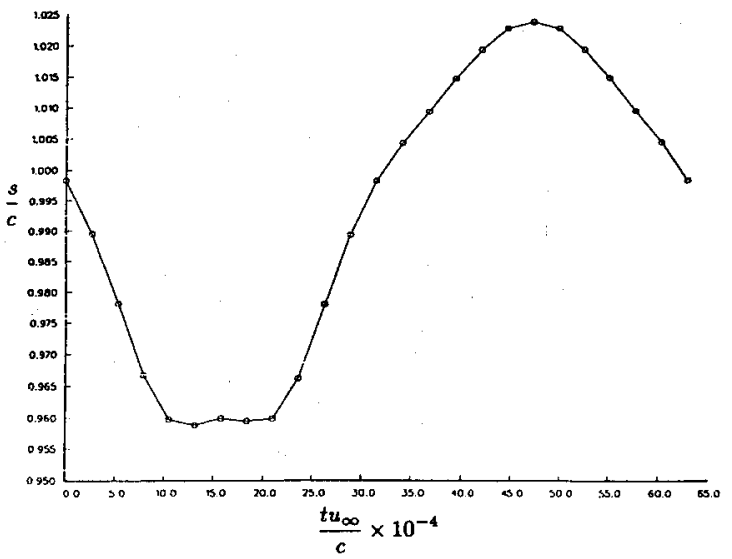

b)

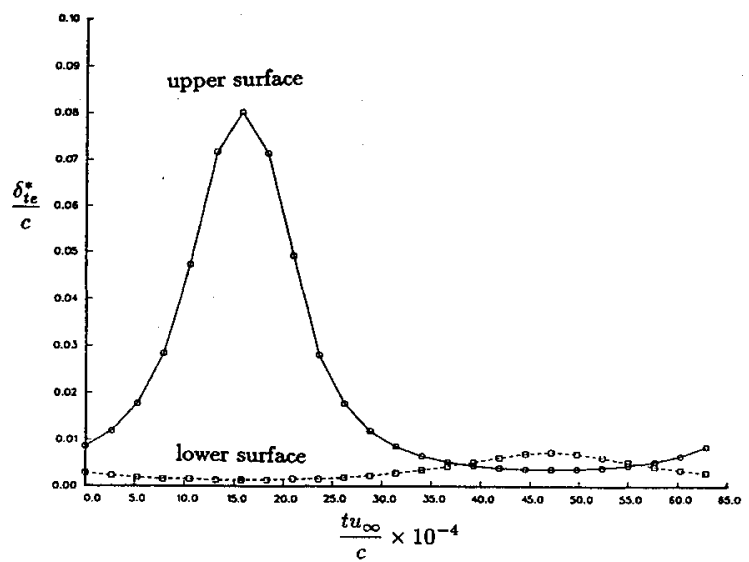

Fig. 5 Calculated results with the quasisteady model for a harmonic motion according to $\mathrm{Eq}$. (7) at $\omega=10^{-5}, R_{c}=2 \times 10^{6}$ : a) locus of the leading-edge stagnation point; b) variation of trailing-edge displacement thickness on the upper and lower surfaces with phase angle.

since time is associated with angle of attack, the same results could be determined from Figs. 2 to 5 .

The quasisteady calculation method was also used to determine the flow over the airfoil when subjected to a ramp-type motion from 0 to $16 \mathrm{deg}$, and, again, the results were identical to those of Figs. 2-4. Figure 6 is presented to show the influence of the wake on the calculation of lift coefficient and confirms that it must be included for angles of attack above around $8 \mathrm{deg}$.

The quantification of the wake effect in the slow-motion cases provides a basis for evaluating the effect in more severe unsteady flows. When the airfoil performs slow movements, the vorticities shed to the wake are weak and the wake, due to viscosity, behaves like a distribution of sinks along the dividing line. Increasing the amplitude and frequency of the unsteady motion increases the strength of the vorticity shed to the wake and the wake of the flow over an airfoil can, therefore, be described as including sources of vorticity and sinks that mix and interact with each other. In the present calculations, the incorporation of the wake involved the assumption that vorticity and sinks are distinct and do not influence each other. This means that the vorticity shed via the potential flow is transported downsteam by the local velocities without the influence of sinks, and the sinks computed from the viscous wake are distributed on the instantaneous wake dividing line, whose location is evaluated without the influence of vorticity. This model satisfies the steady flow condition as the unsteady motion approaches zero and should capture most of the characteristics of the wake as the unsteady motion of the airfoil increases. Some instantaneous wake dividing lines for the Sikorsky airfoil executing a harmonic motion, given by Eq.
(7), at $\omega=0.001,0.01,0.1$, and 0.5 are shown in Fig. 7, and the wake locations for frequencies below 0.1 are very close to those for the steady wake. With $\omega=0.5$, the lines and locations are quite different in the far-wake region but not in the near wake where the wake-viscosity effects are most important.

Figure 8 presents variations of lift coefficient with angle of attack for the same airfoil subject to the same ramp motion and the same Reynolds number but operating at higher pitch rates corresponding to $A=0.01$ and 0.02 . As expected, increasing the value of $A$ decreases the role of viscous effects so

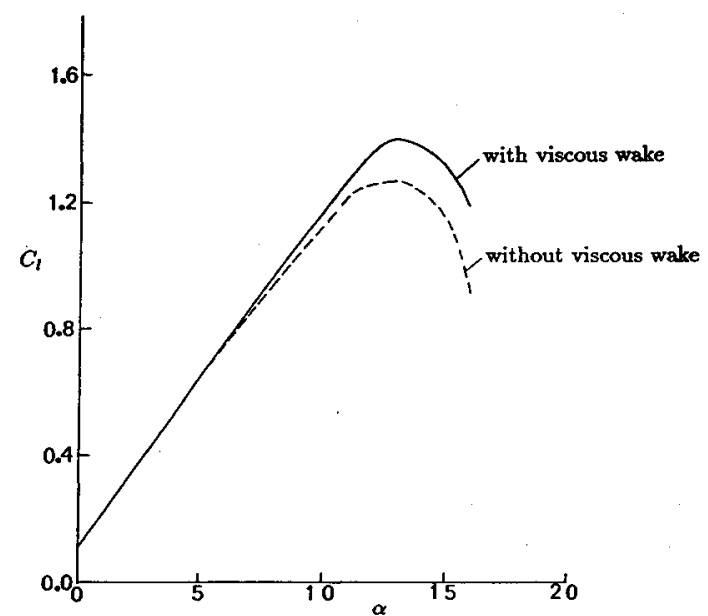

Fig. 6 Effect of viscous wake on lift-coefficient distribution; quasisteady, ramp-type motion with $\alpha$ varying from 0 to $16 \mathrm{deg}$ and with constant pitch rate, $A=10^{-5}, R_{c}=2 \times 10^{6}$.
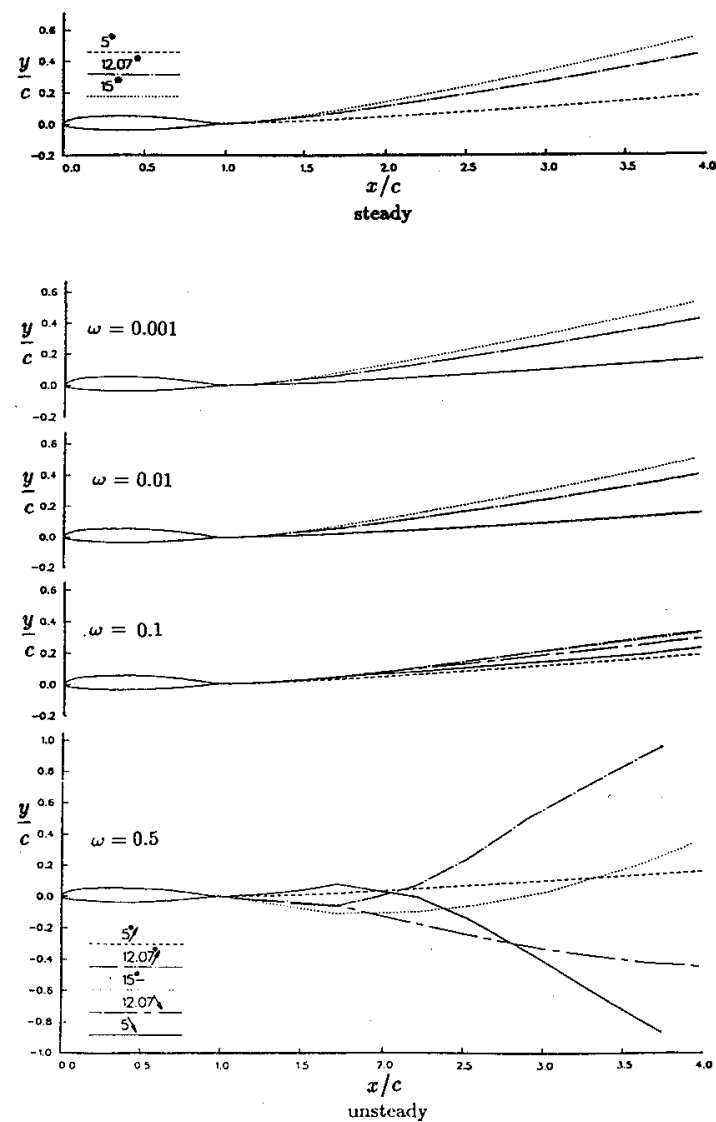

Fig. 7 Comparison of instantaneous wake dividing lines for the Sikorsky airfoil executing a harmonic oscillation according to Eq. (7) at different frequencies with those of steady flow. 
a)
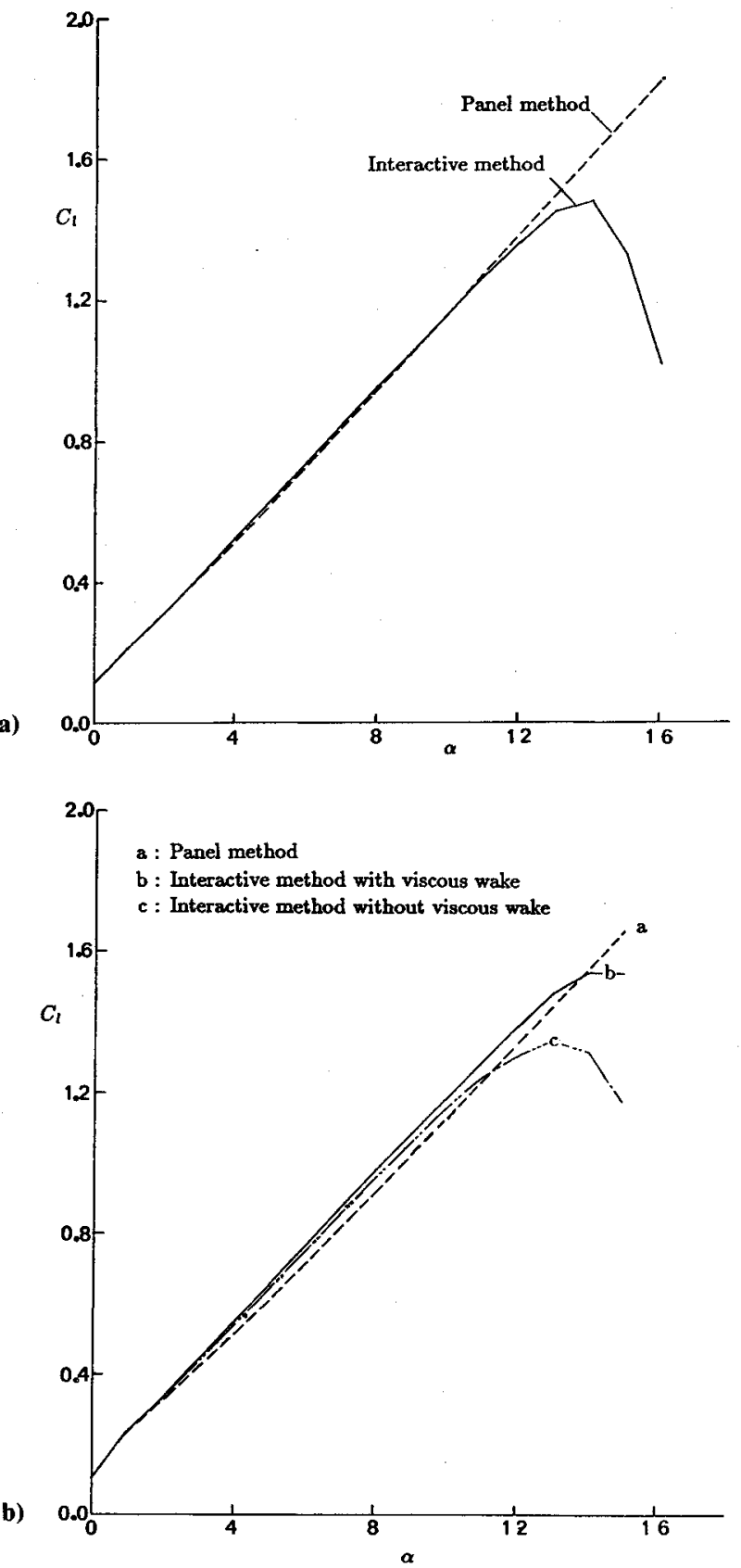

Fig. 8 Variation of lift coefficient with angle of attack; quasisteady, ramp-type motion with constant pitch rate: a) $A=0.01$; b) $A=0.02$.

that, at small angles of attack, the viscous lift coefficient is slightly higher than its inviscid counterpart. The difference, however, diminishes at $\alpha$ around $9 \mathrm{deg}$, after which the viscous lift coefficients become less than the inviscid values, and near the poststall exhibit the behavior of lift coefficients for steady flows. Figure 8b shows that doubling the pitch rate $A=0.02$ enhances the "over-react" behavior of viscous-lift coefficients, and it remains to determine if this results is real or a consequence of the models used in our study. Comparison of Figs. 2, 8a, and $8 \mathrm{~b}$ shows that an increase in pitch rate leads to an increase in maximum lift, probably associated with a delay in stall to higher angle of attack.

\section{Conclusions}

The results presented in this paper are entirely computational and need to be appraised in terms of physical results. They show, however, that the quasisteady computational method works satisfactorily for oscillating and ramp-type mo- tions of an airfoil. The importance of the effects of the viscous layer and of the wake are clearly demonstrated. In light of the recent work for poststall steady flows, it would be interesting and useful to apply the present method to unsteady flows over airfoils at angles of attack much higher than those considered here and to investigate the behavior of the solutions on the airfoil and in the wake in relation to measurements. This will inevitably lead to the need to replace the quasiboundary-layer approach by an interactive scheme in which unsteady boundary-layer equations are solved.

\section{Acknowledgment}

This research was supported under Air Force Office of Scientific Research Contract F49620-88-C-0020.

\section{References}

${ }^{1}$ Carr, L. W., "Progress in Analysis and Prediction of Dynamic Stall," Journal of Aircraft, Vol. 25, No. 1, Jan. 1988, pp. 6-17.

${ }^{2}$ Cebeci, T., Carr, L. W., Khattab, A. A., and Schimke, S. M., "Computational Aspects of Unsteady Flows," Paper No. 8, Proceedings of the AGARD Conference on Unsteady Aerodynamics-Fundamentals and Applications to Aircraft Dynamics, Göttingen, Germany, May 1985.

3 Proceedings of the Fourth Symposium on Numerical and Physical Aspects of Aerodynamic Flows. California State Univ., Long Beach, CA, Jan. 1989.

${ }^{4}$ Cebeci, T., Clark, R. W., Change, K. C., Halsey, N. D., and Lee, K., "Airfoils with Separation and the Resulting Wakes," Journal of Fluid Mechanics, Vol. 173, 1986, pp. 323-347.

${ }^{5}$ Mehta, U., Chang, K. C., and Cebeci, T., "Relative Advantages of Interactive and Thin Navier-Stokes Procedures," Numerical and Physical Aspects of Aerodynamic Flows, III, edited by T. Cebeci, Springer-Verlag, New York, 1986.

${ }^{6}$ Chang, K. C., Alemdaroglu, N., Mehta, U., and Cebeci, T., "Further Comparisons of Interactive Boundary-Layer and Thin-Layer Navier-Stokes Procedures," Journal of Aircraft, Vol. 25, Oct. 1988, pp. 897-903.

${ }^{7}$ Gregory, N., and O'Reilly, C. L., “'Low-Speed Aerodynamics Characteristics of NACA 0012 Airfoil Section, Including the Effects of Upper-Surface Roughness Simulating Hoar Frost," National Physical Laboratory, England, Aero Rept. 1308, 1970.

${ }^{8}$ Cebeci, T., Jau, J., Vitiello, D., and Chang, K. C., "'Prediction of Post-Stall Flows on Airfoils," Numerical and Physical Aspects of Aerodynamic Flows, IV, edited by T. Cebeci, Springer-Verlag, Heidelberg, 1990.

'Reyhner, T. A., and Flügge-Lotz, I., "The Interaction of a Shock Wave With a Laminar Boundary Layer," International Journal of Non-Linear Mechanics, Vol. 3, No. 2, 1968, p. 173-199.

${ }^{10}$ Cebeci, T., Khattab, A. A., and Schimke, S. M., "Separation and Reattachment Near the Leading Edge of a Thin Oscillating Airfoil,", Journal of Fluid Mechanics, Vol. 188, 1988, pp. 253-274.

"Teng, N. G., "The Development of a Computer Code for the Numerical Solution of Unsteady Inviscid, and Incompressible Flow Over an Airfoil," M. S. Thesis, Naval Postgraduate School, Monterey, CA, 1987.

${ }^{12}$ Hess, J. L., and Smith, A. M. O., "Calculation of Potential Flow About Arbitrary Bodies," Progress in Aeronautical Sciences: Guidance and Control, Vol. 8, Pergamon, New York, 1966, pp. 1-138.

${ }^{13}$ Basu, B. C., and Hancock, G. J., "The Unsteady Motion of Two-Dimensional Airfoil in Incompressible Inviscid Flow," Journal of Fluid Mechanics, Vol. 87, No. 1, 1978, pp. 159-178.

${ }^{14}$ Veldman, A. E. P., "New Quasi-Simultaneous Method to Calculate Interacting Boundary Layers," AIAA Journal, Vol. 19, No. 1, 1981, pp. $79-85$.

${ }^{15}$ LeBalleur, J. C., "Couplage Visqueux-Non Visqueux: Methode Numerique et Applications Aux Ecoulements Bidimensionnels Transsonique Set Supersoniques," Le Recherche Aerospatiale, No. $178-2,1978$, pp. 65-76.

${ }^{16}$ Carter, J. E., "A New Boundary-Layer Inviscid Iteraction Technique for Separated Flow," AIAA Paper 75-1450, July 1979.

${ }^{17}$ Michel, R., "Etude de la Transition sur les Profiles D'Aile; Establissement d'un Critere de Determination de Point de Transition et Calcul de la Trainee de Profile Incompressible," ONERA Rept. 1/1578A, 1951.

${ }^{18}$ Lorber, P. F., and Carta, F. O., "Airfoil Dynamic Stall at Constant Pitch Rate and High Reynolds Number," Journal of Aircraft, Vol. 25, No. 6, June 1988, pp. 548-556. 